\title{
Pourquoi il faut actualiser la procréation médicalement assistée
}

\author{
Jürg Schlup
}

Dr, président de la FMH

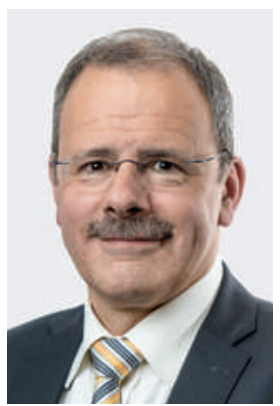

Aujourd'hui en Suisse, un couple sur six a des difficultés à concrétiser son désir d'enfant - un ordre de grandeur qui nous rappelle l'importance d'une actualisation de la procréation médicalement assistée. Afin que ces couples puissent à l'avenir bénéficier d'un accompagnement optimal, il est indispensable d'adapter notre législation par trop restrictive, et aujourd'hui dépassée par le progrès médical.

Contrairement à presque tous les autres pays européens, la Suisse interdit le diagnostic préimplantatoire (DPI). Alors que les partisans de cette interdiction soulignent le risque de sélectionner des soi-disant "existences dignes d'être vécues» ou des «designer babies», les couples concernés se heurtent aux conséquences douloureuses de cette interdiction, dont le risque accru de fausse couche. Du point de vue éthique, le fait que l'interdiction du DPI augmente le nombre d'interruptions de grossesse est particulièrement problématique. La législation actuelle interdit, en effet, de réaliser un diagnostic préimplantatoire sur un ovule fécondé de 5 jours, ce qui pourtant permettrait d'éviter son implantation dans l'utérus en cas de résultat défavorable. Elle autorise

\section{Notre législation restrictive et dépassée par} le progrès médical nécessite une adaptation.

par contre dès la $11^{\mathrm{e}}$ semaine un diagnostic prénatal, et l'interruption de grossesse qui en découlerait, pourtant beaucoup plus traumatisant pour la mère et l'enfant.

La procréation médicalement assistée est réglementée à deux niveaux: d'une part, par l'article 119 de la Constitution fédérale; d'autre part, par la loi sur la procréation médicalement assistée (LPMA) qui en résulte. Même si le DPI est interdit en Suisse au niveau de la loi, la votation de juin sur l'article 119 de la Constitution fédérale constitue une condition indispensable à son autorisation. La réglementation actuelle de la Constitution, selon laquelle «ne peuvent être développés hors du corps de la femme jusqu'au stade d'embryon que le nombre d'ovules humains pouvant être immédiatement implantés», limite dans les faits l'implantation à trois embryons. Comme en "cas normal», seul un parmi six ovules fécondés pourra devenir un enfant viable, et que cette probabilité diminue chez les parents porteurs d'une maladie génétique, le DPI ne peut guère avoir de chances de succès avec une telle limitation. Il est fréquent que les couples concernés ne soient finalement pas en

\section{L'acceptation de la modification constitution-} nelle améliorera la situation des couples qui souffrent de ne pas avoir d'enfant ou qui seraient porteurs d'une maladie génétique.

mesure de donner le jour à un enfant en bonne santé, malgré une procédure laborieuse, lourde et également très coûteuse.

Le projet envisagé de modification constitutionnelle, selon lequel «ne peuvent être développés hors du corps de la femme jusqu'au stade d'embryon que le nombre d'ovules humains nécessaires à la procréation médicalement assistée" permettrait à l'avenir de prévenir beaucoup de souffrances, qui seraient aujourd'hui déjà évitables.

La votation visant l'ajustement de cette demi-phrase dans la Constitution nous permettrait de ne plus priver, pour des raisons uniquement juridiques, les patientes et les patients des progrès de la médecine moderne. En cas d'acceptation de la modification constitutionnelle, le peuple pourrait se prononcer courant 2016 sur les aménagements possibles de la loi sur la procréation médicalement assistée. Mais une chose est certaine: le rejet de la modification constitutionnelle serait une occasion manquée d'améliorer la situation des couples qui souffrent de ne pas avoir d'enfant ou qui seraient porteurs d'une maladie génétique. 